\title{
openheart Detection of unknown atrial fibrillation by prolonged ECG monitoring in an all- comer patient cohort and association with clinical and Holter variables
}

\author{
Muhammad Jawad-UI-Qamar, ${ }^{1,2}$ Winnie Chua, ${ }^{1}$ Yanish Purmah, ${ }^{1,2}$ \\ Mohammad Nawaz, ${ }^{2}$ Chetan Varma, ${ }^{2}$ Russell Davis, ${ }^{2}$ Abdul Maher, ${ }^{2}$ \\ Larissa Fabritz, ${ }^{1,2}$ Paulus Kirchhof (D) 1,2,3
}

To cite: Jawad-UI-Qamar M, Chua W, Purmah Y, et al. Detection of unknown atrial fibrillation by prolonged ECG monitoring in an all-comer patient cohort and association with clinical and Holter variables. Open Heart 2020;7:e001151. doi:10.1136/ openhrt-2019-001151

The work for this paper was performed at the Department of Cardiology, Sandwell and West Birmingham Hospitals NHS Trust, UK and the Institute of Cardiovascular Sciences, University of Birmingham, Birmingham, UK.

Received 8 August 2019 Accepted 12 February 2020

A) Check for updates

(C) Author(s) (or their employer(s)) 2020. Re-use permitted under CC BY. Published by BMJ.

${ }^{1}$ Institute of Cardiovascular Sciences, University of Birmingham, Birmingham, UK ${ }^{2}$ Department of Cardiology, Sandwell and West Birmingham Hospitals NHS Trust, West Bromwich, UK

${ }^{3}$ University Heart and Vascular Center UKE Hamburg, Hamburg, Germany

Correspondence to Professor Paulus Kirchhof; $p$. kirchhof@bham.ac.uk

\section{ABSTRACT}

Objectives Prolonged ECG monitoring is clinically useful to detect unknown atrial fibrillation (AF) in stroke survivors. The diagnostic yield of prolonged ECG monitoring in other patient populations is less well characterised. We therefore studied the diagnostic yield of prolonged Holter ECG monitoring for $\mathrm{AF}$ in an unselected patient cohort referred from primary care or seen in a teaching hospital. Methods We analysed consecutive 7-day ECG recordings in unselected patients referred from different medical specialities and assessed AF detection rates by indication, age and comorbidities.

Results Seven-day Holter ECGs (median monitoring 127.5 hours, IQR 116 to 152) were recorded in 476 patients (mean age 54.6 (SD 17.0) years, $55.9 \%$ female) without previously known $A F$, requested to evaluate palpitations $(n=241)$, syncope $(n=99)$, stroke or transient ischaemic attack $(n=75)$, dizziness $(n=29)$ or episodic chest pain $(n=32)$. AF was newly detected in $42 / 476$ (8.8\%) patients. Oral anticoagulation was initiated in 40/42 $(95.2 \%)$ patients with newly detected AF. Multivariate logistic regression, adjusted for age, sex and monitoring duration found four clinical parameters to be associated with newly detected AF: hypertension $\mathrm{OR}=2.54$, (1.08 to 8.61) (adjusted OR $(95 \% \mathrm{Cl})$ ), $\mathrm{p}=0.034$; previous stroke or TIA OR=4.14 (1.81 to 13.01), $p=0.001$; left-sided valvular heart disease $\mathrm{OR}=5.07$ (2.48 to 18.70), $\mathrm{p}<0.001$ and palpitations $\mathrm{OR}=2.86$, (1.33 to 10.44$), p=0.015$

Conclusions Open multispeciality access to prolonged ECG monitoring, for example, as part of integrated, cross-sector AF care, can accelerate diagnosis of AF and increase adequate use of oral anticoagulation, especially in older and symptomatic patients with comorbidities.

\section{INTRODUCTION}

Atrial fibrillation (AF), a common cause of stroke and cardiovascular death, often remains undetected until a severe complication occurs, such as an ischaemic stroke. ${ }^{12}$ Timely diagnosis of AF is a major clinical need with immediate therapeutic consequences in patients at high stroke risk who would benefit from oral anticoagulation. ${ }^{34}$ Systematic ECG

\section{Key questions}

What is already known about this subject?

- Systematic ECG monitoring is recommended for detection of atrial fibrillation (AF) in patients with ischaemic stroke.

What does this study add?

- Detection of AF through in an all-comer patient population through systematic ECG monitoring is not previously reported.

How might this impact on clinical practice?

- Judicious use of Holter ECG monitoring for new AF detection especially in the presence of clinical factors increasing the chances of AF detection could have significant diagnostic and therapeutic implications.

monitoring improves detection of undiagnosed paroxysmal AF in survivors of a stroke or transient ischaemic attack (TIA), detecting $\mathrm{AF}$ in around $4 \%$ of unselected stroke survivors using 3-day Holter monitoring ${ }^{56}$ and in up to $25 \%$ of selected patients with cryptogenic stroke using implantable monitors. ${ }^{37}$ Recent guidelines recommend at least 72 hours of ECG monitoring in stroke survivors to detect silent $\mathrm{AF} .{ }^{18}$ The diagnostic yield of prolonged ECG monitoring in other groups of patients at risk of $\mathrm{AF}$ has not been studied systematically. We therefore quantified $\mathrm{AF}$ detection rates of 7-day Holter ECG monitoring in an unselected cohort of consecutive patients referred for such monitoring, and identified factors that are associated with detection of unknown AF by multivariate regression.

\section{METHODS}

\section{Patients and procedures}

We analysed 584 consecutive 7-day Holter ECG recordings performed in adult patients 


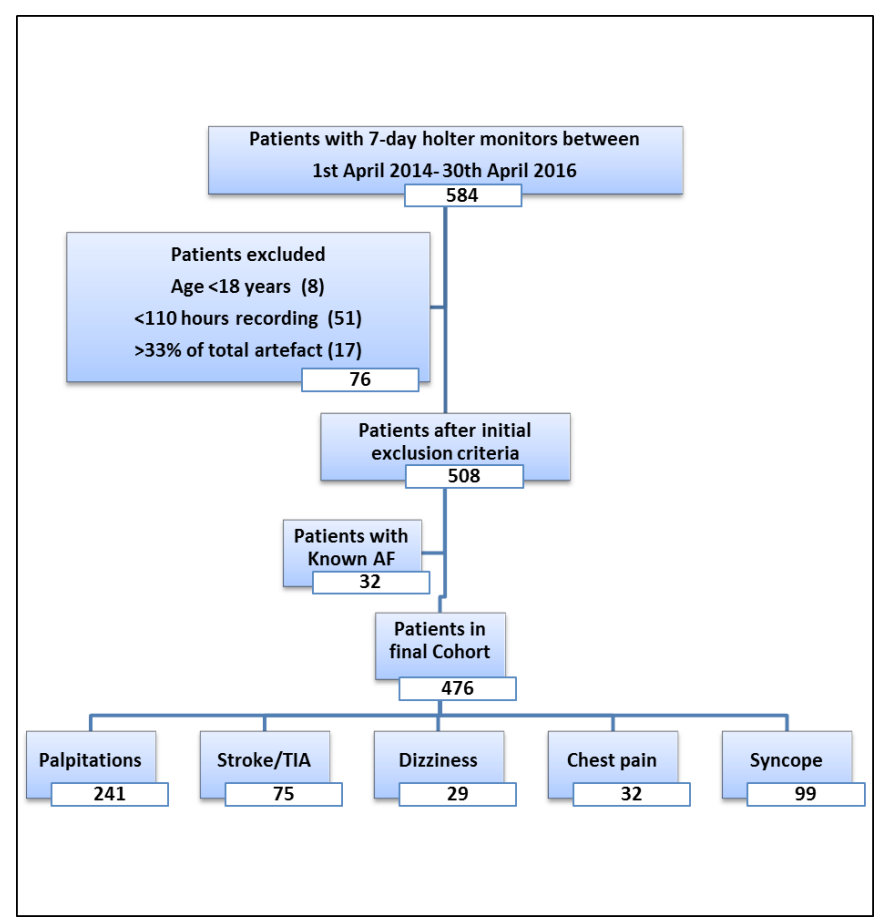

Figure 1 Overview of the patient population. AF, atrial fibrillation; TIA, transient ischaemic attack.

(>18 years) in Sandwell and West Birmingham Hospitals (SWBH) NHS Trust from $1^{\text {st }}$ April 2014 to $30^{\text {th }}$ April 2016. Open access 7-day Holter monitoring is available to hospital physicians and general practitioners in participating practices in our region for various indications. All recordings made through Spacelabs Healthcare modular digital Holter recorder, with Lifecard CF, were initially analysed and reported by trained cardiac physiologists using Pathfinder 1.71 running on Sentinel server V.17.1.1 (Spacelabs, UK). All analyses were subsequently reviewed by two cardiology physicians (JQ, YP). We did not find any discrepancy between the initial analysis and its review for AF detection and any Holter ECG parameter of interest. We excluded 108 patients with known $\mathrm{AF}$, those $<18$ years of age and recordings with $<110$ hours analysable monitoring duration. The remaining 476 recordings constituted our primary analysis data set (figure 1). Clinical information was verified by three researchers (JQ YP, $\mathrm{MN}$ ) from hospital records. This analysis was approved by the clinical effectiveness department at SWBH NHS Trust as part of our ongoing quality improvement programme (QIP SG323).

\section{Definitions}

The study outcome, AF, was defined as chaotic atrial arrhythmia with loss of $\mathrm{p}$ wave activity and irregular $\mathrm{RR}$ interval lasting for at least $30 \mathrm{~s}$. We defined atrial or supraventricular ectopic as narrow QRS complex similar to sinus beat with different or absent $\mathrm{p}$ wave and $<80 \%$ of prevailing RR interval. Supraventricular ectopic (SVE) runs were defined as regular or irregular runs of three or more consecutive atrial ectopics lasting for less than 30 s. Sinus pause was defined as increase in RR interval due to delay in $\mathrm{p}$ wave activity resulting in a pause $>2 \mathrm{~s}$. We categorised significant atrial ectopy as $\geq 50$ atrial ectopics/day and significant sinus pauses as $\geq 10$ sinus pauses/day based on cut-offs calculated through receiver operating characteristic (ROC) curve. All comorbidities were taken as previously documented through records from primary care or specialist clinics. Valvular disease was taken as moderateto-severe left (mitral or aortic) valvular disease, stenosis or regurgitation confirmed on prior echocardiography.

\section{Statistical analysis}

Normality was assessed by inspecting the frequency distribution curves. For normally distributed variables we reported mean and SD, for other parameters median and IQR. Categorical variables were compared using $\chi^{2}$ tests and continuous variables using independent t-test or Mann-Whitney $U$ test as appropriate. The $p$ values of $<0.05$ were considered statistically significant. The cutoffs for continuous variables were calculated through the Youden's index (maximum sensitivity and minimum 1-specificity) obtained from the receiver operating characteristics (ROC) curve. The univariate relationship to the outcome was quantified using logistic regression. A multivariate logistic regression model through enter method was fitted using a combination of various clinical variables which includes hypertension, left-sided moderate/ severe valvular disease, previous cerebrovascular accident and palpitations which were chosen due to their clinical relevance. The derived model was adjusted for sex, age and duration of recording. The model was internally validated using bootstrapping to produce an optimismadjusted model. We assessed the bootstrapped model's performance by quantifying the C-statistic (equivalent to area under the ROC curve). All the statistical analyses were performed using SPSS V.24 (IBM).

\section{RESULTS \\ Clinical characteristics of the patient population and AF detection}

Mean age of the study population was 56 years, $56 \%$ were female, and $40 \%$ were older than 60 years (table 1 ). AF was diagnosed in $42 / 476$ patients $(8.8 \%)$ of which three had new AF throughout the recording and the remaining had paroxysmal AF. Median number of AF episodes was 4 (IQR 1.75 to 5.25), total duration of longest AF episode was $28 \mathrm{~min}$ (IQR 7.9 to 180.4 ) and median duration of longest episode was $731 \mathrm{~min}$ (IQR 3.9 to 115.1 ), respectively. After detection of AF, 40/42 (95.2\%) patients were started on oral anticoagulation.

Patients with AF detected were older (mean 69.8 years, SD 69.8) with no significant difference in sex and $31 / 42(73.8 \%)$ patients with $\mathrm{AF}$ were age 60 years or more (figure 2). The AF group had more comorbidities such as hypertension, coronary artery disease, history of stroke or transient ischaemic attack, moderate-to-severe left-sided valvular disease and palpitations. There was no difference seen for diabetes and hypercholesterolaemia. 
Arrhythmias and sudden death

\begin{tabular}{|c|c|c|c|c|}
\hline Variable & Total $(n=476)$ & AF detected $(n=42)$ & AF not detected $(n=434)$ & $\mathrm{p}$ value \\
\hline \multicolumn{5}{|l|}{ Clinical parameters } \\
\hline Age, years, mean (SD) & $56.4(17.0)$ & $69.8(12.2)$ & $53.1(16.7)$ & $<0.001$ \\
\hline Female, n (\%) & $266(56)$ & $22(52)$ & $244(56)$ & 0.632 \\
\hline Hypertension, n (\%) & $173(36.3)$ & $32(76.1)$ & $141(32.4)$ & $<0.001$ \\
\hline Diabetes, n (\%) & 78 (16.3) & $11(26.2)$ & $67(18)$ & 0.072 \\
\hline Coronary artery disease, $\mathrm{n}(\%)$ & $102(21.4)$ & $17(40.4)$ & $85(19.5)$ & 0.002 \\
\hline Hypercholesterolaemia, n (\%) & $177(37.1)$ & $17(40.4)$ & $160(36.8)$ & 0.644 \\
\hline History of stroke or transient ischaemic attack, $\mathrm{n}(\%)$ & $111(23.3)$ & $23(54.7)$ & $88(20.2)$ & $<0.001$ \\
\hline Valvular disease, $\mathrm{n}(\%)$ & $72(15.1)$ & $18(42.8)$ & $54(12.4)$ & $<0.001$ \\
\hline Palpitations, n (\%) & $241(50.63)$ & $32(76.19)$ & 209 (48.15) & 0.001 \\
\hline \multicolumn{5}{|l|}{ Holter parameters } \\
\hline Recording, hours, median (IQR) & 127.5 (116 to 152$)$ & $153.4(133.7$ to 166.0$)$ & $124.0(115.0$ to 127.5$)$ & 0.001 \\
\hline Mean heart rate, bpm, mean (SD) & $79.9(13.3)$ & $104.7(13.0)$ & $77.5(11.2)$ & 0.001 \\
\hline Max heart rate, bpm, mean (SD) & $139.1(18.2)$ & $144.9(12.4)$ & $138.5(18.6)$ & 0.004 \\
\hline Min heart rate, bpm, mean (SD) & $49.2(8.3)$ & $50.4(10.5)$ & $49.0(8.1)$ & 0.417 \\
\hline$\geq 10$ sinus pauses/day (\%) & $77(16.1)$ & $26(61.9)$ & $51(11.7)$ & $<0.001$ \\
\hline$\geq 50$ atrial ectopics/day (\%) & $156(32.7)$ & $36(85.7)$ & $120(27.6)$ & $<0.001$ \\
\hline$\geq 500$ ventricular ectopics/recording (\%) & $443(93)$ & $42(100)$ & $401(92.3)$ & 0.064 \\
\hline Supraventricular ectopic (SVE) runs, (\%) & $70(14.7)$ & $26(61.9)$ & $44(10.1)$ & $<0.001$ \\
\hline Longest atrial run, mean (SD) & $8.7(4.0)$ & $10.9(2.8)$ & $8.1(4.0)$ & $<0.001$ \\
\hline
\end{tabular}

Data presented as number, $\mathrm{n}($ percentage,\%) for categorical variables, mean (SD) for parametric continuous variables, and median (IQR) for nonparametric continuous variables. $p$ value is significant if $<0.05$.

$\mathrm{AF}$, atrial fibrillation; bpm, beats per minute; SD, Standard Deviation.

The recording duration in AF group was longer, median (IQR), 153.4 hours (133.7 to 166.0) versus non-AF group 124.0 hours (115.0 to 127.5). Moreover there were more sinus pauses, atrial ectopics and SVE runs. If present, SVE runs were longer in the AF group. AF was more often found in stroke survivors $(7 / 75,9.3 \%)$ and in patients with palpitations $(32 / 241,13.3 \%)$ than in patients with dizziness $(2 / 29,6.8 \%)$, syncope $(2 / 99,2 \%)$ or chest pain $(1 / 32,3.1 \%)$ (figure 3$)$. The source of referral for the test did not affect AF detection (cardiology 8.7\% (19/218),

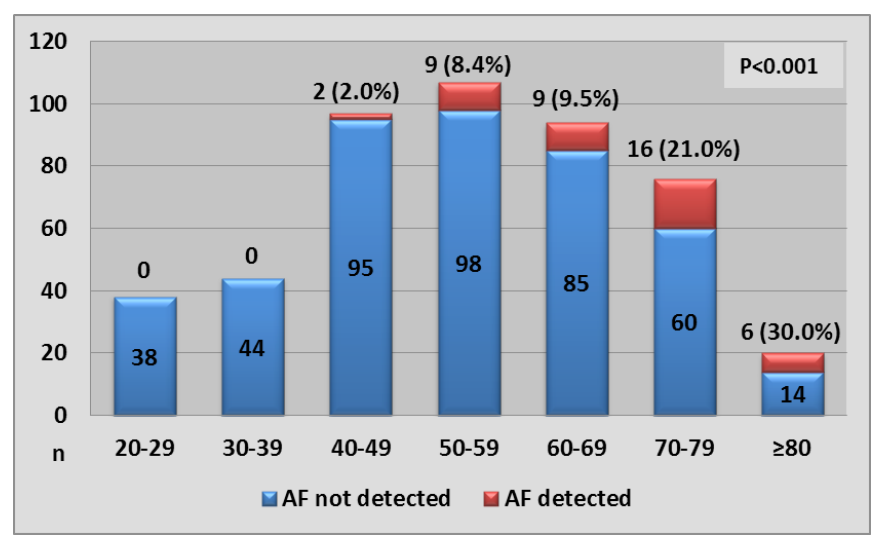

Figure 2 Number of patients with and without newly detected AF split by age deciles. Percentage of patients with newly detected $A F$ is given above the bars for each decile. $A F$, atrial fibrillation. general medicine 9\% (19/222) and primary care $8.6 \%$ $(4 / 46))$.

\section{Other relevant findings}

Other medically relevant findings were second degree atrioventricular (AV) block $(10 / 474,2.1 \%)$, third degree AV block $(2 / 474,0.4 \%)$ and non-sustained ventricular tachycardia $(12 / 474,2.5 \%)$. These were all detected in patients who underwent monitoring for dizziness. Artefact burden in the final cohort was 3.3\% (SD 3.5)

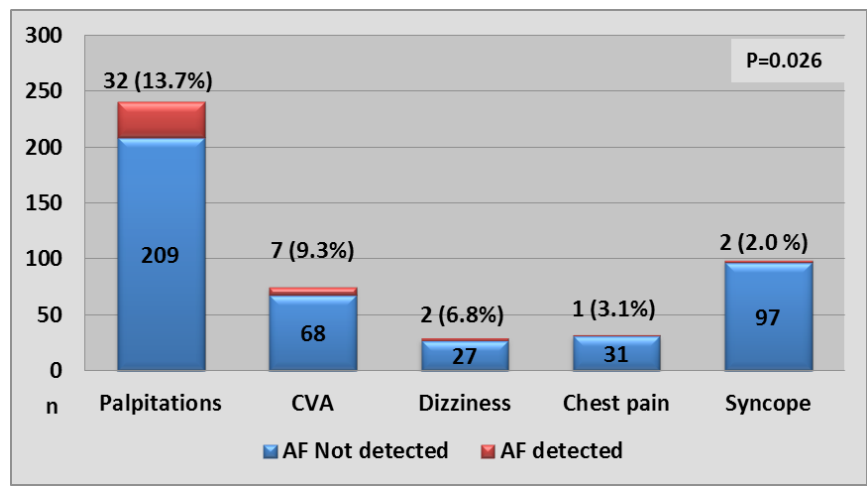

Figure 3 Number of patients with and without newly detected AF by underlying conditions to justify the 7-day Holter monitor. AF, atrial fibrillation; CVA, cerebrovascular accident. 


\begin{tabular}{|c|c|c|c|c|}
\hline \multirow[b]{2}{*}{ Variables } & \multirow[b]{2}{*}{ OR } & \multicolumn{2}{|c|}{$\begin{array}{l}95 \% \text { Confidence } \\
\text { Intervals (Cl) }\end{array}$} & \multirow[b]{2}{*}{$p$ value } \\
\hline & & $\begin{array}{l}\text { Lower } \\
\text { limit }\end{array}$ & $\begin{array}{l}\text { Upper } \\
\text { limit }\end{array}$ & \\
\hline \multicolumn{5}{|c|}{ Univariate logistic regression analysis } \\
\hline Age (per year increase) & 1.08 & 1.06 & 1.11 & $<0.001$ \\
\hline Hypertension & 6.65 & 3.17 & 13.90 & $<0.001$ \\
\hline Diabetes & 1.94 & 0.93 & 4.07 & 0.077 \\
\hline Coronary artery disease & 2.79 & 1.44 & 5.40 & 0.002 \\
\hline Hypercholesterolaemia & 1.16 & 0.61 & 2.22 & 0.644 \\
\hline Valvular disease & 5.27 & 2.68 & 10.35 & $<0.001$ \\
\hline Previous CVA/TIA & 4.76 & 2.48 & 9.12 & $<0.001$ \\
\hline Palpitations & 3.44 & 1.65 & 7.18 & 0.001 \\
\hline $\begin{array}{l}\text { Recording duration (per } \\
\text { hour increase) }\end{array}$ & 1.05 & 1.03 & 1.07 & $<0.001$ \\
\hline$\geq 10$ sinus pauses/day & 12.2 & 6.13 & 24.27 & $<0.001$ \\
\hline$\geq 50$ AE/day & 15.70 & 6.45 & 38.21 & $<0.001$ \\
\hline Presence of SVE runs & 14.40 & 7.17 & 28.90 & $<0.001$ \\
\hline \multicolumn{5}{|c|}{ Variables (bootstrapped) } \\
\hline \multicolumn{5}{|c|}{ Multivariate logistic regression analysis } \\
\hline Hypertension & 2.54 & 1.08 & 8.61 & 0.034 \\
\hline Previous CVA & 4.14 & 1.81 & 13.01 & 0.001 \\
\hline Valvular disease & 5.07 & 2.48 & 18.70 & $<0.001$ \\
\hline Palpitations & 2.86 & 1.33 & 10.44 & 0.015 \\
\hline
\end{tabular}

$\mathrm{AE}$, atrial ectopic; AF, atrial fibrillation; CVA, cerebrovascular accident; SAE, significant atrial ectopy; SVE, supraventricular ectopic; TIA, transient ischaemic attack.

\section{Clinical parameters associated with AF}

Univariately, AF was more likely to be detected in patients who are older (OR 10.85, 95\% CI 10.56 to 11.15 for each 10 year increase in age), and in those presenting with palpitations (OR 3.44, 95\% CI 1.65 to 7.18, $\mathrm{p}=0.001$ ), have hypertension (OR 6.65, 95\% CI 3.17 to $13.90, \mathrm{p}<0.001$ ), coronary artery disease (OR 2.79, 95\% CI 1.44 to 5.40, $\mathrm{p}=0.003$ ), history of ischaemic strokes or TIA (OR 4.76, $95 \%$ CI 2.48 to $9.12, \mathrm{p}<0.001$ ), or moderate-to-severe left-sided valve disease (OR 5.27, 95\% CI 2.68 to 10.35 , $\mathrm{p}<0.001$ ).

\section{Holter parameters associated with AF}

$\mathrm{AF}$ detection was also univariately associated with longer ECG monitoring (OR 1.05, 95\% CI 1.03 to 1.07 for each additional hour of monitoring), sinus pauses (OR 12.2, $95 \%$ CI 6.13 to $24.27, \mathrm{p}<0.001$ ), significant atrial ectopics (OR 15.70, 95\% CI 6.45 to $38.21, \mathrm{p}<0.001$ ) and presence of SVE runs (OR 14.4, 95\% CI 7.17 to $28.90, \mathrm{p}<0.001$, table 2).

Multivariate regression, performed through enter method identified four variables to be significantly associated with undetected AF: Previous stroke or transient ischaemic attack bootstrapped OR=4.14 (95\% CI 1.81 to 13.01), $\mathrm{p}=0.001$; left-sided moderate-to-severe valvular

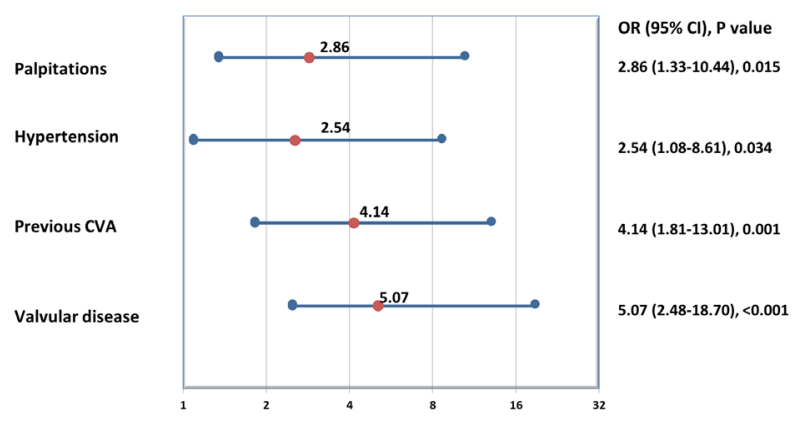

Figure 4 Individual predictors of newly detected AF by 7-day Holter, shown as hazard ratio and $95 \%$ confidence interval. CVA,cerebrovascular accident.

disease OR=5.07 (95\% CI 2.48 to 18.70), $\mathrm{p}<0.001$; palpitations $\mathrm{OR}=2.86$, (95\% CI 1.33 to 10.44$), \mathrm{p}=0.015$ and hypertension $\mathrm{OR}=2.54$, (95\% CI 1.08 to 8.61 ) $\mathrm{p}=0.034$; table 2 , figure 4 . The model was adjusted for age, sex and duration of recording.

The area under the curve (C statistic) for our multivariate model was 0.91 (95\% CI 0.87 to 0.95$)$ with individual C-statistics for each variable mentioned in figure 5 , table 3 .

\section{DISCUSSION}

We found that 7-day ECG monitoring detected unknown $\mathrm{AF}$ in $9 \%$ of an unselected cohort presenting with various symptoms. Almost all patients were initiated on oral anticoagulation following AF detection. The AF detection rates of $9.3 \%$ in stroke survivors is comparable to $\mathrm{AF}$ rates in IDEAS $\left(4.3 \%, 72\right.$ hours monitoring, ${ }^{5}$ FIND-AF $(12.5 \%$, 10 days monitoring $)^{9}$ and FIND-AF ${ }_{\text {randomised }}(14 \%) .{ }^{6}$ The data supports the wider use of systematic ECG monitoring to allow early initiation of therapy for AF, particularly stroke prevention, for example, as part of an integrated

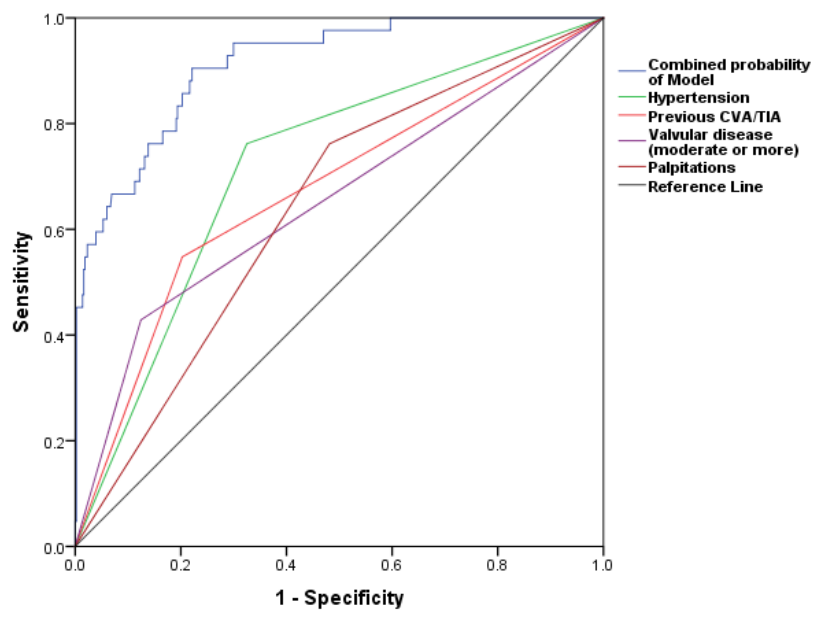

Figure 5 Receiver operating characteristic (ROC) curves for the different AF prediction models. See text for details.CVA, cerebrovascular accident; TIA, transient ischaemic attack. 


\begin{tabular}{lllll}
\hline Table 3 & Area under the curve values and Cls \\
\cline { 1 - 4 } & \multicolumn{4}{c}{$\begin{array}{l}\text { 95\% Confidence } \\
\text { Intervals (CI) }\end{array}$} \\
\cline { 2 - 4 } Variable(s) & Area & $\begin{array}{l}\text { Lower } \\
\text { bound }\end{array}$ & $\begin{array}{l}\text { Upper } \\
\text { bound }\end{array}$ & p value \\
\hline Combined model & 0.912 & 0.870 & 0.954 & $<0.001$ \\
Hypertension & 0.719 & 0.639 & 0.798 & $<0.001$ \\
Previous CVA & 0.672 & 0.580 & 0.765 & $<0.001$ \\
Valvular disease & 0.652 & 0.555 & 0.749 & $<0.001$ \\
\hline Palpitations & 0.640 & 0.558 & 0.723 & 0.003 \\
\hline
\end{tabular}

CVA, cerebrovascular accident.

approach to AF care. External validation of our findings is desirable.

\section{Factors associated with newly detected AF}

Patients with $\mathrm{AF}$ were older (table 1, figures 2 and 4) than those without $\mathrm{AF}$, similar to other reports ${ }^{1011}$ and consistent with the known finding that older age is associated with prevalent $\mathrm{AF}^{12}{ }^{13}$ We found that each decade increase in age increased the odds of detecting AF by 10.8. In addition, previous history of stroke and left-sided valvular heart disease was associated with silent $\mathrm{AF}$ in univariate analysis which is comparable to prior reports. ${ }^{14} 15$ In our study palpitations were the symptom that was most frequently associated with underlying $\mathrm{AF}$ (figure 4). Our analysis supports some prior reports associating palpitations with $\mathrm{AF}^{16}$ while others found a more ephemeral nature of presenting symptoms. ${ }^{17}$ As expected, longer monitoring duration increased $\mathrm{AF}$ detection. ${ }^{18} \mathrm{In}$ addition, excessive supraventricular ectopic activity was associated with unknown AF (table 2, figure 4), comparable to prior analyses. ${ }^{19-21}$ Sinus node disease, identified by sinus pauses, ${ }^{22}$ has been linked to underlying $\mathrm{AF}^{23}{ }^{24}$ Although, sinus pauses were not part of our multivariate model, but there is evidence to suggest that it could be associated with unknown $\mathrm{AF}$, confirming findings from one prior study. ${ }^{25}$ We suggest further ECG monitoring, beyond 7 days, to look for $\mathrm{AF}$ in clinically high risk patients as identified by our multivariate model, especially, if they have evidence of significant atrial ectopy or sinus pauses on prior monitoring.

\section{Open access ECG monitoring - an alternative approach to increase detection of unknown AF?}

Current guidelines recommend pulse palpation followed by an ECG in patients with an irregular pulse in populations $>65$ years to detect $\mathrm{AF}^{18}{ }^{8}$ Pulse palpation can be replaced by a blood pressure machine. ${ }^{26}{ }^{27}$ Systematic prolonged ECG monitoring is recommended in stroke survivors. In addition, community-based ECG screening programmes using patient-operated devices are able to identify patients with silent AF, especially when elderly populations are screened, for example, those aged 75 years or more. ${ }^{28-30}$ Such population-based screening programmes require a specific infrastructure that is currently confined to a research environment or not available at all. Our study suggests that open access to prolonged ECG monitoring has a high diagnostic yield in patients with various symptoms, opening up an alternative approach to increase the detection of unknown AF. Open access to ECG monitoring has the advantage of using existing health infrastructure, but is limited to populations with symptoms.

\section{Limitations}

Our findings will need validation in other, external patient cohorts. Although the number of patients in our study was reasonable for a busy teaching hospital however more patients are required for further studies especially to assess Holter ECG variables with incident AF. The number of patients also had an effect on the relatively wide CIs for the calculated ORs. AF group had longer duration of monitoring that could introduce bias. To rectify this we adjusted our multivariate variable for duration of recording. Moreover, older patient usually have higher incidence of comorbidities which may be one reason why the multivariate model strongly predicted AF.

\section{CONCLUSION}

Seven-day Holter ECG monitoring detects unknown $\mathrm{AF}$ in around $9 \%$ of patients with various symptoms in an open access, 'all-comer' setting, suggesting that this is a useful diagnostic approach to enhance detection of unknown AF. Risk factor analysis for paroxysmal AF and systematic evaluations of treatment and outcomes after AF screening are needed to define populations for screening silent $\mathrm{AF}$. Comparable $\mathrm{AF}$ detection rates between cardiology and primary care also supports provision of ECG monitoring using open-access services across various healthcare sectors.

Acknowledgements We thank the cardiology diagnostic services department, cardiology clinicians and clinical effectiveness department at Sandwell and West Birmingham Hospitals NHS trust, for their support in conducting this study.

Funding This study was supported by the clinical effectiveness programme of SWBH NHS trust. Further support came from European Union (grant agreement 633196 (CATCH ME) and by British Heart Foundation (AA/18/2/34218). PK has received research support from EU, BHF, Leducq Foundation, Medical Research Council (UK) and German Centre for Cardiovascular Research, from several drug and device companies active in $\mathrm{AF}$, and has received honoraria from several such companies.

Competing interests PK and LF are listed as inventors on two patents held by University of Birmingham (WO 2015140571, WO 2016012783). RD and AM have received honoraria for delivering educational lectures from manufacturers of anticoagulant medications.

Patient consent for publication Not required.

Provenance and peer review Not commissioned; externally peer reviewed.

Data availability statement Data are available upon reasonable request subject to permission from organisation.

Open access This is an open access article distributed in accordance with the Creative Commons Attribution 4.0 Unported (CC BY 4.0) license, which permits others to copy, redistribute, remix, transform and build upon this work for any purpose, provided the original work is properly cited, a link to the licence is given, and indication of whether changes were made. See: https://creativecommons.org/ licenses/by/4.0/. 
ORCID iD

Paulus Kirchhof http://orcid.org/0000-0002-1881-0197

\section{REFERENCES}

1 Freedman B, Camm J, Calkins $\mathrm{H}$, et al. Screening for atrial fibrillation: a report of the AF-SCREEN international collaboration. Circulation 2017;135:1851-67.

2 Kirchhof P, Breithardt G, Bax J, et al. A roadmap to improve the quality of atrial fibrillation management: proceedings from the fifth atrial fibrillation Network/European heart rhythm association consensus conference. Europace 2016;18:37-50.

3 Gladstone DJ, Spring M, Dorian P, et al. Atrial fibrillation in patients with cryptogenic stroke. N Engl J Med 2014;370:2467-77.

4 Hart RG, Pearce LA, Aguilar MI. Meta-analysis: antithrombotic therapy to prevent stroke in patients who have nonvalvular atrial fibrillation. Ann Intern Med 2007;146:857-67.

5 Grond M, Jauss M, Hamann G, et al. Improved detection of silent atrial fibrillation using 72-hour Holter ECG in patients with ischemic stroke: a prospective multicenter cohort study. Stroke 2013;44:3357-64.

6 Wachter R, Gröschel K, Gelbrich G, et al. Holter-electrocardiogrammonitoring in patients with acute ischaemic stroke (Find$\mathrm{AF}_{\text {RANDOMISED }}$ ): an open-label randomised $_{\text {controlled trial. Lancet Neurol }}$ 2017;16:282-90.

7 Sanna T, Diener H-C, Passman RS, et al. Cryptogenic stroke and underlying atrial fibrillation. N Engl J Med 2014;370:2478-86.

8 Kirchhof P, Benussi S, Kotecha D, et al. 2016 ESC guidelines for the management of atrial fibrillation developed in collaboration with EACTS. Eur Heart J 2016;37:2893-962.

9 Stahrenberg R, Weber-Krüger M, Seegers J, et al. Enhanced detection of paroxysmal atrial fibrillation by early and prolonged continuous Holter monitoring in patients with cerebral ischemia presenting in sinus rhythm. Stroke 2010;41:2884-8.

10 Suissa L, Bertora D, Lachaud S, et al. Score for the targeting of atrial fibrillation (STAF): a new approach to the detection of atria fibrillation in the secondary prevention of ischemic stroke. Stroke 2009;40:2866-8

11 Verdecchia P, Reboldi G, Gattobigio R, et al. Atrial fibrillation in hypertension: predictors and outcome. Hypertension 2003;41:218-23.

12 Leitch JW, Thomson D, Baird DK, et al. The importance of age as a predictor of atrial fibrillation and flutter after coronary artery bypass grafting. J Thorac Cardiovasc Surg 1990;100:338-42.

13 Aranki SF, Shaw DP, Adams DH, et al. Predictors of atrial fibrillation after coronary artery surgery. Current trends and impact on hospital resources. Circulation 1996;94:390-7.

14 Poli S, Diedler J, Härtig F, et al. Insertable cardiac monitors after cryptogenic stroke - a risk factor based approach to enhance the detection rate for paroxysmal atrial fibrillation. Eur J Neurol 2016;23:375-81.
15 Christensen LM, Krieger DW, Højberg S, et al. Paroxysmal atrial fibrillation occurs often in cryptogenic ischaemic stroke. Final results from the SURPRISE study. Eur J Neurol 2014;21:884-9.

16 Hansson A, Madsen-Härdig B, Olsson SB. Arrhythmia-provoking factors and symptoms at the onset of paroxysmal atrial fibrillation: a study based on interviews with 100 patients seeking Hospital assistance. BMC Cardiovasc Disord 2004;4:13.

17 Smeets JLRM. Paroxysmal atrial fibrillation: why patients experience different symptoms from the same arrhythmia? Neth J Med 2005;63:154-5

18 Kirchhof P, Auricchio A, Bax J, et al. Outcome parameters for trials in atrial fibrillation: Executive summary: recommendations from a consensus conference organized by the German atrial fibrillation competence network (AFNET) and the European heart rhythm association (EHRA). Eur Heart J 2007;28:2803-17.

19 Waktare JE, Hnatkova K, Sopher SM, et al. The role of atrial ectopics in initiating paroxysmal atrial fibrillation. Eur Heart $J$ 2001;22:333-9.

20 Perez MV, Dewey FE, Marcus R, et al. Electrocardiographic predictors of atrial fibrillation. Am Heart J 2009;158:622-8.

21 Weber-Krüger M, Gröschel K, Mende M, et al. Excessive supraventricular ectopic activity is indicative of paroxysmal atrial fibrillation in patients with cerebral ischemia. PLoS One 2013;8:e67602.

22 Rodriguez RD, Schocken DD. Update on sick sinus syndrome, a cardiac disorder of aging. Geriatrics 1990;45:26-36.

23 Alt E, Lehmann G. Stroke and atrial fibrillation in sick sinus syndrome. Heart 1997;77:495-7.

24 Zdrojewicz Z, Iwankiewicz G, Rutkowski J, et al. [Paroxysmal atrial fibrillation as an integral part of sick (damaged) sinus syndrome]. Wiad Lek 1989;42:13-19.

25 Soeki T, Matsuura T, Tobiume T, et al. Clinical, electrocardiographic, and echocardiographic parameter combination predicts the onset of atrial fibrillation. Circ J 2018;82:2253-8.

26 Chan P-H, Wong C-K, Pun L, et al. Head-To-Head comparison of the AliveCor heart monitor and Microlife WatchBP office AFIB for atrial fibrillation screening in a primary care setting. Circulation 2017;135:110-2

27 Chan P-H, Wong C-K, Pun L, et al. Diagnostic performance of an automatic blood pressure measurement device, Microlife WatchBP home a, for atrial fibrillation screening in a real-world primary care setting. BMJ Open 2017;7:e013685.

28 Svennberg E, Engdahl J, Al-Khalili F, et al. Mass screening for untreated atrial fibrillation: the STROKESTOP study. Circulation 2015;131:2176-84

29 Engdahl J, Svennberg E, Friberg L, et al. Stepwise mass screening for atrial fibrillation using $\mathrm{N}$-terminal pro $\mathrm{B}$-type natriuretic peptide: the STROKESTOP II study design. Europace 2017;19:297-302.

30 Chan N-Y, Choy C-C. Screening for atrial fibrillation in 13122 Hong Kong citizens with smartphone electrocardiogram. Heart 2017;103:24-31. 